\title{
Phase structure of excited baryonic matter in the relativistic mean field theory
}

\author{
B. M. Waldhauser, J. Theis, J. A. Maruhn, H. Stöcker, and W. Greiner \\ Institut für Theoretische Physik der Johann-Wolfgang-Goethe Universität, D-6000 Frankfurt am Main, \\ Federal Republic of Germany \\ (Received 15 December 1986)
}

\begin{abstract}
We analyze the phase structure of the nonlinear mean-field meson theory of baryonic matter (nucleons plus delta resonances). Depending on the choice of the coupling constants, we find three physically distinct phase transitions in this theory: a nucleonic liquid-gas transition in the low temperature, $T_{c}<20 \mathrm{MeV}$, low density, $\rho \simeq 0.5 \rho_{0}$, regime, a high-temperature $(T \simeq 150 \mathrm{MeV})$ finite density transition from a gas of massive hadrons to a nearly massless baryon, antibaryon plasma, and, third, a strong phase transition from the nucleonic fluid to a resonance-dominated "deltamatter" isomer at $\rho>2 \rho_{0}$ and $T_{c}<50 \mathrm{MeV}$. All three phase transitions are of first order. It is shown that the occurrence of these different phase transitions depends critically on the coupling constants. Since the production of pions also depends strongly on the coupling constants, it is seen that the equation of state cannot be derived unambiguously from pion data.
\end{abstract}

\section{INTRODUCTION}

In a preceding paper $^{1}$ we investigated the phase structure of the linear self-consistent relativistic field theoretical model of baryonic matter. ${ }^{2}$ We have found that for coupling constants which reproduce the observed binding energy and density of nuclear matter a phase transition occurs for baryon density zero, i.e., for vanishing chemical potential $(\mu=0)$, from a gas of massive nucleons to a plasma of nearly massless nucleons and antinucleons. This sudden change was signalled by a peak in the specific heat. This transition is due to the rapid increase of the attractive scalar field at $T \simeq 200 \mathrm{MeV}$, which initiates the drop in the effective nucleon mass. In the present work we extend our previous investigation of the phase structure by explicitly including nonlinear terms $^{3}$ in the scalar interaction which allows a more realistic description of nuclear compressibilities and effective masses than the linear model. Furthermore, isobaric resonances ${ }^{4-9}$ are included. In this approach the properties of baryonic matter depend on six parameters $C_{s}, C_{v}, B, C, \alpha=g_{v}(\Delta) / g_{v}(\mathrm{~N})$, and $\beta=g_{s}(\Delta) / g_{s}(\mathrm{~N})$. The strength of the dimensionless coupling constants $\alpha$ and $\beta$ of the delta resonance to the vector and scalar mesons is not known a priori.

We show in the present paper that the plasma phase transition for $\mu=0$ (Ref. 1) in the linear model does also appear at finite baryochemical potentials in the nonlinear theory. A liquid-gas phase transition is also observed, but at low density and low temperature. We then show that for certain values of $\alpha$ and $\beta$ density isomers occur which are compatible with known nuclear ground-state properties: A phase transition from a nucleonic fluid to resonance-dominated "delta matter" at $\rho \geq 2 \rho_{0}$ with a critical temperature $T_{c} \leq 50 \mathrm{MeV}$ is observed. We also show that the plasma transition of Ref. 1 is not directly related to such a hypothetical density isomeric state.

\section{THE NONLINEAR RELATIVISTIC MESON MEAN-FIELD MODEL OF STRONGLY INTERACTING MATTER}

The nucleon field $\Psi_{\mathrm{N}}$ and delta field $\Psi_{\Delta}$ interact in the present approach ${ }^{2,3}$ through a scalar field $\varphi$ and a vector field $V_{\mu}$, while the pion and rho meson mean fields vanish in symmetric nuclear matter in the mean-field theory. The simplest nonderivative coupling of baryons to meson fields is given by the Lagrange density: ${ }^{2,3}$

$$
\begin{aligned}
L= & \bar{\Psi}_{\mathrm{N}}\left(i \gamma_{\mu} \partial^{\mu}-m_{\mathrm{N}}\right) \Psi_{\mathrm{N}}+\bar{\Psi}_{\Delta}\left(i \gamma_{\mu} \partial^{\mu}-m_{\Delta}\right) \Psi_{\Delta} \\
& -\frac{1}{2} \partial_{\mu} \varphi \partial^{\mu} \varphi-U(\varphi)-\frac{1}{4} F_{\mu v} F^{\mu \nu}-\frac{1}{2} m_{v}^{2} V_{\mu} V^{\mu} \\
& -g_{s}(\mathrm{~N}) \bar{\Psi}_{\mathrm{N}} \Psi_{\mathrm{N}} \varphi-g_{s}(\Delta) \bar{\Psi}_{\Delta} \Psi_{\Delta} \varphi \\
& -g_{v}(\mathrm{~N}) \bar{\Psi}_{\mathrm{N}} \gamma_{\mu} \Psi_{\mathrm{N}} V^{\mu}-g_{v}(\Delta) \bar{\Psi}_{\Delta} \gamma_{\mu} \Psi_{\Delta} V^{\mu}
\end{aligned}
$$

where the field tensor is defined as

$$
F_{\mu v}=\frac{\partial V_{v}}{\partial x_{\mu}}-\frac{\partial V_{\mu}}{\partial x_{v}}
$$

and the nonlinear scalar potential ${ }^{3}$ is written as

$$
U(\varphi)=\frac{1}{2} m_{s}^{2} \varphi^{2}+\frac{1}{3} b \varphi^{3}+\frac{1}{4} c \varphi^{4} .
$$

For symmetric, infinite isotropic nuclear matter one derives in the mean field approach the following equations of motion:

$$
\begin{aligned}
& \left\{i \gamma_{\mu} \partial^{\mu}-\left[m_{\mathrm{N}}+g_{s}(\mathrm{~N}) \varphi_{0}\right]-g_{v}(\mathrm{~N}) \gamma_{0} V^{0}\right\} \Psi_{\mathrm{N}}=0, \\
& \left\{i \gamma_{\mu} \partial^{\mu}-\left[m_{\Delta}+g_{s}(\Delta) \varphi_{0}\right]-g_{v}(\Delta) \gamma_{0} V^{0}\right\} \Psi_{\Delta}=0, \\
& m_{s}^{2} \varphi_{0}+b \varphi_{0}^{2}+c \varphi_{0}^{3}=-g_{s}(\mathrm{~N}) \rho_{s}(\mathrm{~N})-g_{s}(\Delta) \rho_{s}(\Delta), \\
& m_{v}^{2} V_{0}=+g_{v}(\mathrm{~N}) \rho_{v}(\mathrm{~N})+g_{v}(\Delta) \rho_{v}(\Delta) .
\end{aligned}
$$

Here $V_{0}$ is the zero component of the repulsive vector field $V_{\mu}$. The effective baryon masses $m^{*}(\mathbf{N})$ and $m^{*}(\Delta)$, 
respectively, are defined by

$$
\begin{aligned}
& m^{*}(\mathrm{~N})=m_{\mathrm{N}}+g_{s}(\mathrm{~N}) \varphi_{0}, \\
& m^{*}(\Delta)=m_{\Delta}+g_{s}(\Delta) \varphi_{0},
\end{aligned}
$$

with $m_{\mathrm{N}}=939 \mathrm{MeV}$ and $m_{\Delta}=1232 \mathrm{MeV}$. Using Eq. (4a), we write for the effective delta mass

$$
m^{*}(\Delta)=m_{\Delta}+\frac{g_{s}(\Delta)}{g_{s}(\mathrm{~N})}\left[m^{*}(\mathrm{~N})-m_{\mathrm{N}}\right] .
$$

For finite temperature baryonic matter the scalar densities are given by

$\rho_{s}(i)=\frac{\gamma(i)}{(2 \pi)^{3}} \int_{0}^{\infty} d^{3} k \frac{m^{*}(i)}{\left[k^{2}+m^{* 2}(i)\right]^{1 / 2}}\left[n_{i}(T)+\bar{n}_{i}(T)\right]$,

while the vector densities are derived as

$$
\rho_{v}(i)=\frac{\gamma(i)}{(2 \pi)^{3}} \int_{0}^{\infty} d^{3} k\left[n_{i}(T)-\bar{n}_{i}(T)\right] .
$$

Thus the baryon density is the sum of the nucleon and delta vector densities:

$$
\rho_{B}=\rho_{v}(\mathrm{~N})+\alpha \rho_{v}(\Delta) .
$$

Here the $n_{i}(T), \bar{n}_{i}(T)$ stand for the Fermi-Dirac distributions for baryons and antibaryons, respectively,

$$
\begin{aligned}
& \left.n_{i}(T)=\left[\exp \left(\left\{k^{2}+m^{* 2}(i)\right]^{1 / 2}-v\right\} / T\right)+1\right]^{-1}, \\
& \left.\bar{n}_{i}(T)=\left[\exp \left(\left\{k^{2}+m^{* 2}(i)\right]^{1 / 2}+v\right\} / T\right)+1\right]^{-1} .
\end{aligned}
$$

The abbreviations $i=\mathrm{N}, \Delta$, and $\gamma(i)=4,16$ correspond to spin-isospin- $\frac{1}{2}$ and spin-isospin- $\frac{3}{2}$ particles, respectively. The effective chemical potential is defined as $v=\mu-g_{v} V_{0}=\mu-\rho_{B} g_{v}^{2} / m_{v}^{2}$. The energy density and the pressure are given by

$\epsilon=\frac{1}{2} \frac{g_{v}^{2}}{m_{v}^{2}} \rho_{B}^{2}+U(\varphi)+\sum_{i} \frac{\gamma(i)}{(2 \pi)^{3}} \int_{0}^{\infty} d^{3} k\left[k^{2}+m^{* 2}(i)\right]^{1 / 2}$

$$
\times\left[n_{i}(T)+\bar{n}_{i}(T)\right],
$$

$$
\begin{aligned}
& P= \frac{1}{2} \frac{g_{v}^{2}}{m_{v}^{2}} \rho_{B}^{2}-U(\varphi) \\
&+\frac{1}{3} \sum_{i} \frac{\gamma(i)}{(2 \pi)^{3}} \int_{0}^{\infty} d^{3} k \frac{k^{2}}{\left[k^{2}+m^{* 2}(i)\right]^{1 / 2}} \\
& \\
& \quad \times\left[n_{i}(T)+\bar{n}_{i}(T)\right] .
\end{aligned}
$$

The nucleon and delta effective mass $m_{\mathrm{N}}^{*}$ and $m_{\Delta}^{*}$, respectively, have to satisfy the self-consistency relation for the scalar meson field:

$$
\begin{gathered}
m^{*}(\mathrm{~N})=m_{\mathrm{N}}-C_{s}^{2}\left\{\rho_{s}^{\mathrm{N}}+\beta \rho_{s}^{\Delta}+B\left[m^{*}(\mathrm{~N})-m_{\mathrm{N}}\right]^{2}\right. \\
\left.+C\left[m^{*}(\mathrm{~N})-m_{\mathrm{N}}\right]^{3}\right\} \\
m^{*}(\Delta)=m_{\Delta}+\beta\left[m^{*}(\mathrm{~N})-m_{\mathrm{N}}\right] .
\end{gathered}
$$

The relevant parameters of the model are the dimensionless coupling constants

$C_{s}=\frac{g_{s} m_{\mathrm{N}}}{m_{s}}, \quad C_{v}=\frac{g_{v} m_{\mathrm{N}}}{m_{v}}, \quad B=\frac{b}{g_{s}^{3} m_{\mathrm{N}}}, \quad C=\frac{c}{g_{s}^{4}}$,

$\alpha$ and $\beta$.

The first four coupling constants can be determined from the four known ground-state properties for infinite nuclear matter: saturation density $\rho_{0}=0.15-0.17 / \mathrm{fm}^{3}$, binding energy per nucleon $E / A \simeq-16 \mathrm{MeV}$, compressibility $K=200-300 \mathrm{MeV}$, and effective nucleon mass $m^{*} / m \simeq 0.7$. In the following we use the values obtained by Boguta $^{3}$ from a fit to finite nuclei: $C_{s}^{2}=246.0$, $C_{v}^{2}=156.3, B=-1.8 E-3$ and $C=+2.87 E-4$. They correspond to $\rho_{0}=0.17 / \mathrm{fm}^{3}, E / A=-15.84 \mathrm{MeV}$, and $m^{*} / m=0.678$. Note that while Boguta ${ }^{3,5}$ and Nakai and Takagi ${ }^{6}$ claimed a compressibility of $290 \mathrm{MeV}$, we find $K \simeq 344 \mathrm{MeV}$.

There is no information available about the delta coupling constants $\alpha$ and $\beta$.

\section{CHOICE OF THE DELTA COUPLING CONSTANTS}

On the basis of "quark counting" arguments the corresponding constants were chosen equal in Ref. 4,

$$
g_{s \mathrm{~N}}=g_{s \Delta}
$$

and

$g_{v N}=g_{v \Delta}$.

Figure 1 shows $m^{*}(\mu)$ for various sets of $\alpha$ and $\beta$. The choice above ( $\alpha=\beta=1)$ leads to negative effective nucleon mass as one can see in Fig. 1. At large densities the delta has a small positive effective mass, while the effective mass of the nucleon becomes negative at about $\mu=2 \mathrm{GeV}$.

The problem is that for an effective mass equal to zero the energy gap between particles and antiparticles van-

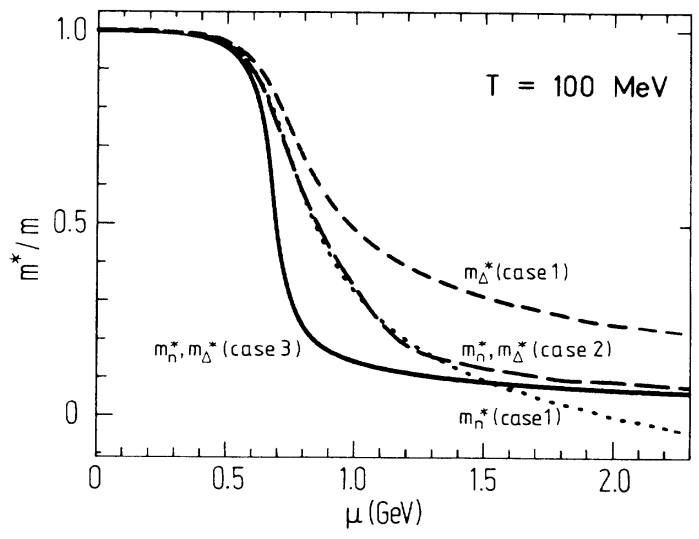

FIG. 1. Effective mass vs the chemical potential $\mu$ for $T=100$ $\mathrm{MeV}$. One can see the influence of the three different sets of coupling constants, Eqs. (12)-(14). For Eq. (12) (case 1) we got two curves, one for the delta mass (dashed) and one for the nucleon mass (dotted). For Eq. (13) (case 2, dashed-dotted) and 14 (case 3, solid line) the masses have the same ratio. 
ishes. That means that spontaneous particle-antiparticle pair production can happen abundantly. This unpleasant behavior one could overcome if the SU(6) symmetry is considered. If the SU(6) symmetry is exact for baryons, then we would be forced to use the same coupling constant for the baryon decuplet and the baryon octet, just as in Ref. 4. but the mass splitting of the multiplets shows that the SU(6) symmetry is not exactly fulfilled. Therefore one could also assume that the coupling coefficients show a splitting similar to that of the mass splitting: ${ }^{9}$

$$
\frac{g_{s \Delta}}{g_{s \mathrm{~N}}}=\frac{g_{v \Delta}}{g_{v \mathrm{~N}}}=\frac{m_{\Delta}}{m_{\mathrm{N}}}
$$

For this choice (Fig. 1) the effective mass ratios $m^{*}(i) / m(i)$ are equal and positive. But one should stress that symmetry arguments can give only tentative indications to choose these ratios, since the theory deals with effective mesons.

A similar result can be obtained with the set of coupling constants studied in Ref. 5, where $\alpha=1$ and $\beta$ was varied between 1.2 and 1.5. These different choices will be studied in the following chapter. The main result is that both effective masses are positive for finite temperature and chemical potential (Fig. 1) and smaller than for the previous choice of the delta coupling constants. In the next section we show that for the baryonic plasma $\left(\mu=\rho_{B}=0\right)$ the value of the scalar coupling to the delta resonance is determined by the restriction of positive effective masses:

$$
\frac{g_{s \Delta}}{g_{s \mathrm{~N}}}=\frac{m_{\Delta}}{m_{\mathrm{N}}} \simeq 1.31
$$

For that case both effective masses will converge to zero from above for infinite temperature. ${ }^{8}$ These considerations apply only to the scalar coupling, because the baryonic plasma does not contain any information about the vector coupling.

Let us now consider the consequences of the different

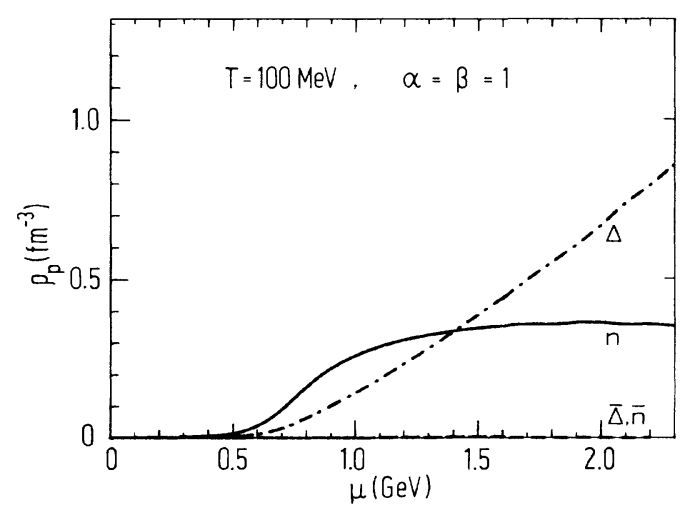

FIG. 2. The particle densities vs the chemical potential $\mu$. The solid line corresponds to the nucleon density, the dasheddotted line to the deltas, while the dotted line represents the antinucleons and the dashed line stands for the antideltas. This figure is connected to Eq. (12).

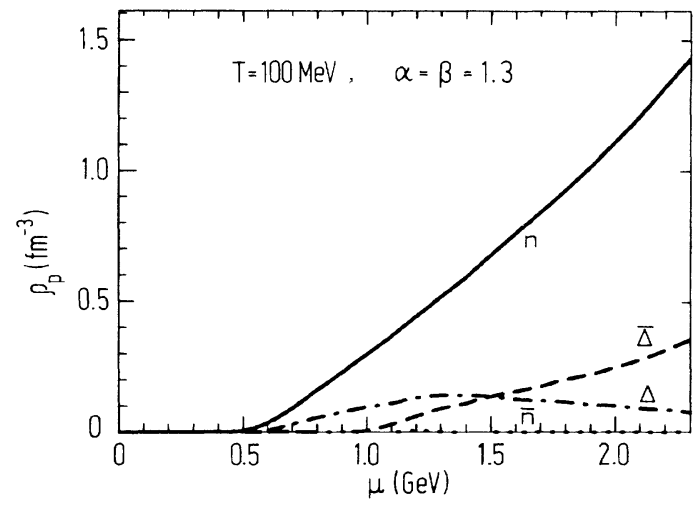

FIG. 3. Same as Fig. 2, except that this figure is connected to Eq. (13).

choices in some detail (Figs. 2-4). We have plotted the particle density of nucleons, antinucleons, deltas, and antideltas versus the chemical potential $\mu$. The choice of Garpman et al. (Ref. 4) shows a clear delta dominance with fewer nucleons and negligible antiparticles (Fig. 2). The second choice with mass dependent scalar and vector coupling ${ }^{9}$ predicts a strong suppression of the deltas (Fig. 3 ). This is due to the strong vector coupling. The third choice with mass dependent scalar and equal vector coupling $^{5,6,8}$ yields delta dominated matter with suppressed nucleons and negligible antiparticle contributions. Here negative effective masses do not occur (Fig. 4). These three possibilities show that the delta abundance is strongly dependent on the coupling constants of the delta resonances to the scalar and vector fields. This result renders the proposed method of using the pion $(\Delta)$ yields to extract the nuclear equation of state from data ${ }^{10,11}$ virtually useless for the mean field equation of state. In this paper we will consider mainly the third choice in more detail.

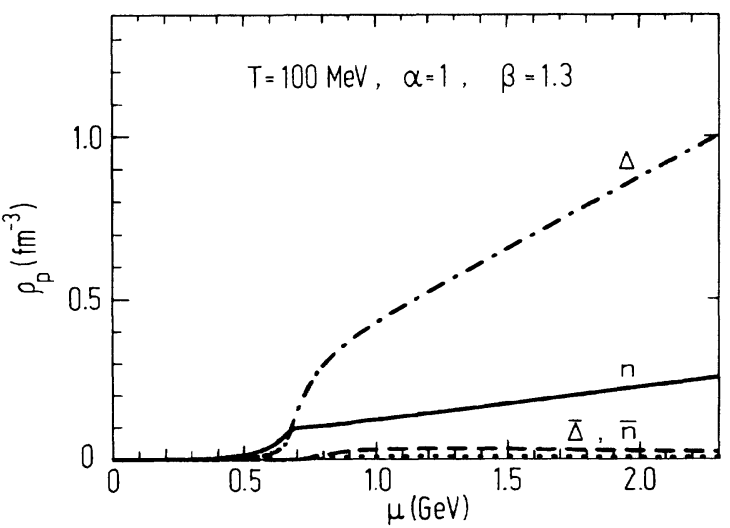

FIG. 4. Same as Fig. 2, except that this figure stands for the set of Eq. (14). 


\section{THREE PHASE TRANSITIONS IN HOT HADRONIC MATTER}

\section{A. Critical phenomena at finite baryochemical potential and densities}

Let us study the phase structure of nuclear matter in analogy to Ref. 1, but at finite baryochemical potential: Figure 5 shows the "equation of state" at $T=0 \mathrm{MeV}$, i.e., the binding energy per nucleon versus the density, for different values of the scalar coupling constant $\beta$ $(\beta=1.31-1.35)$ and the vector coupling constant $\alpha$ $(\alpha=1.0-1.31)$. For $\alpha=1.0$ and $\beta>1.2$, a secondary minimum is obtained at densities $\rho_{B} \geq 2 \rho_{0}$. For $\beta=1.5$ the second minimum is actually lower than the ground state (below $-50 \mathrm{MeV})$. For $\beta \approx m(\Delta) / m(\mathrm{~N})(\alpha=1.0$ and $\beta=1.35)$, a secondary minimum develops at $E / A \cong+4 \mathrm{MeV}$ and $\rho \cong 3 \rho_{0}$. The reason for this behavior is the rapid increase of the delta production at $2 \rho_{0}$

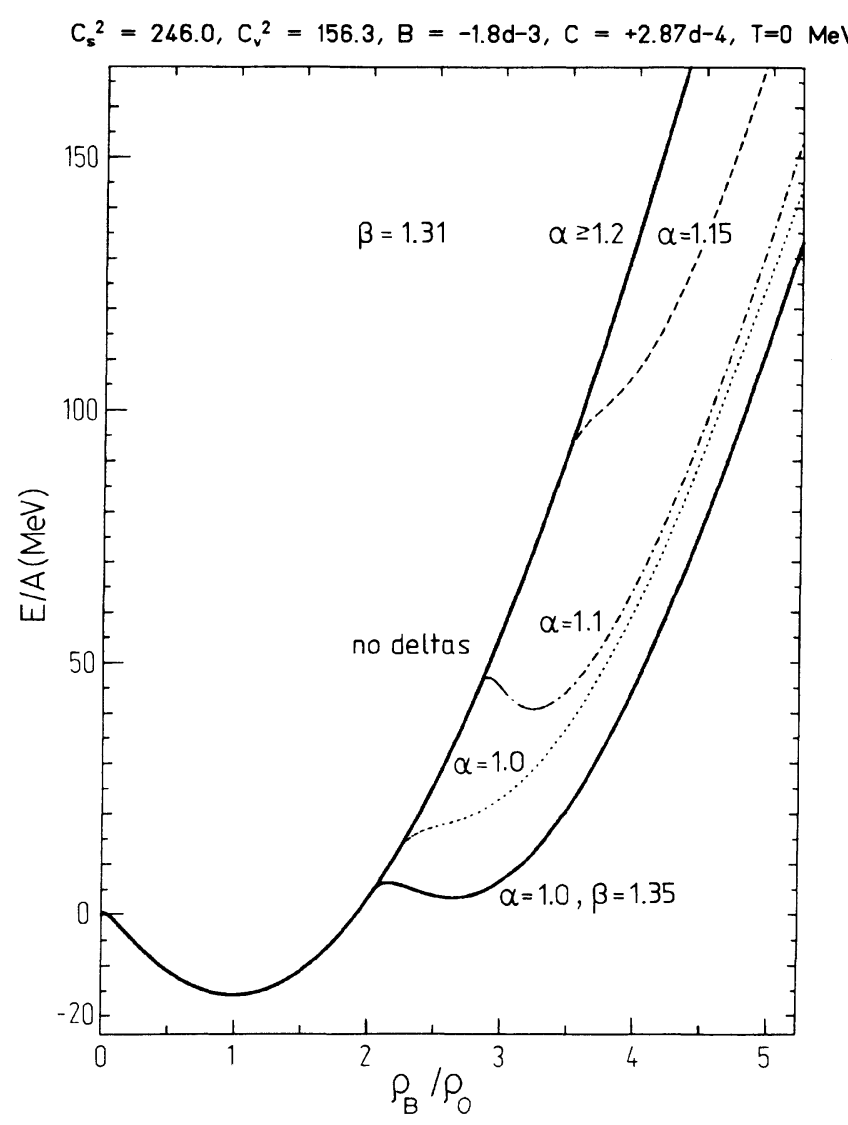

FIG. 5. Equation of state: Binding energy per nucleon vs the baryon density $\rho_{B} / \rho_{0}$ for $\alpha \geq 1.0$ and $\beta=1.31,1.35$ and vanishing temperature $T=0 \mathrm{MeV}$. The left curve (solid) is the nucleonic curve without any delta distribution, but is also valid for $\beta=1.31$ and $\alpha \geq 1.2$. The second (dashed), third (dasheddotted), and fourth (dotted) curves are plotted for $\beta=1.31$ and $\alpha=1.15,1.1$, and $\alpha=1.0$, respectively. For decreasing vector coupling strength $\alpha$ the binding energy decreases and a real minimum is only reached for $\alpha=1.1$. The fifth (solid) curve is the only one with $\beta=1.35(\alpha=1.0)$.

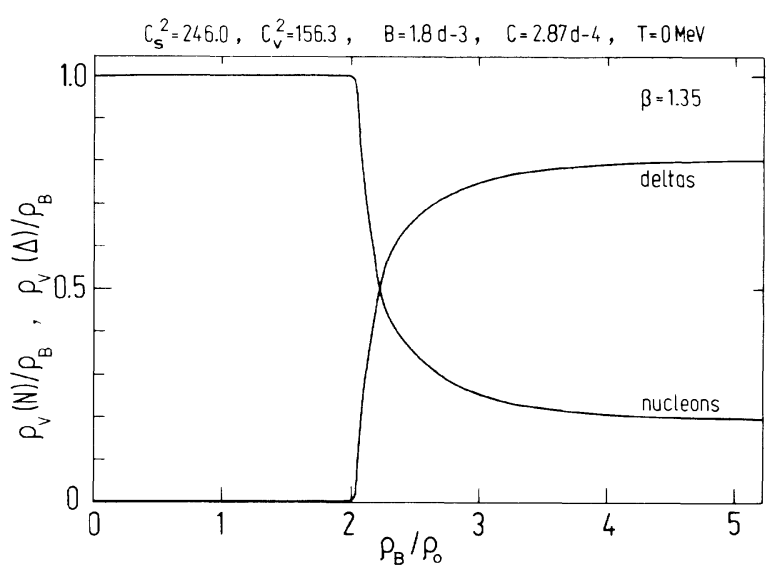

FIG. 6. Particle density of deltas and nucleons vs the baryon density. $\beta$ is chosen to be 1.35 , that is, the density isomeric case.

(Fig. 6). This reflects the strong attraction of the deltas by the scalar field, which results in a lowering of the delta continuum states below the Fermi surface of the nucleons. Equilibrium is reached when $80 \%$ of the baryons are in the deltas (with degeneracy $\gamma=16$ ) and $20 \%$ are in the nucleons $(\gamma=4)$. But also the vector coupling constant $\alpha$ has a great influence on the equation of state. If we fix the scalar coupling constant to $\beta=1.31$ and vary only $\alpha$, the minimum of the density isomer lies higher in energy and vanishes completely for $\alpha>1.15$. Then the delta resonances do not occur at moderate densities. The left curve in Fig. 5 is not only valid for this choice but also for the normal nucleonic equation of state.

Hence we can conclude in agreement with Fig. 5 that in addition to the ground state properties there is a great influence of the delta coupling constants on the high density behavior (Ref. 5). The delta resonances play the most important role for the pion production in heavy ion collisions from $0 \mathrm{MeV}$ to $2 \mathrm{GeV} /$ nucleon bombarding energy. ${ }^{12}$ The present result means that the equation of state cannot be unambiguously derived from the pion data as was expected before. ${ }^{10,11,13}$

For finite temperatures (Fig. 7) we can see a similar behavior: As the temperature increases, so does the energy per nucleon. For $T>20 \mathrm{MeV}$ the system is unbound and for $T=50 \mathrm{MeV}$ the two minima have the same depth. ${ }^{5}$ While the normal nucleonic minimum vanishes, more and more deltas are produced. For $T=100 \mathrm{MeV}$ there is finally only one broad delta dominated minimum in the isotherm. This behavior is due to the smearing effect of the temperature on the Fermi level (compare Fig. 8). Therefore the delta abundance increases much more smoothly, but starts already at zero baryon density with a value above $0.2 \rho_{B}$.

The velocity of sound tends towards the velocity of light for high densities and/or high temperatures as can be seen in Fig. 9: The isotherms of $P$ tend towards the causality limit $P=\epsilon$. That means that $\partial P / \partial \epsilon=c_{s}^{2}$ converges to 1 from below. The second velocity is therefore predicted to be always smaller than the light velocity. In the same picture, two phase transitions can be observed. 


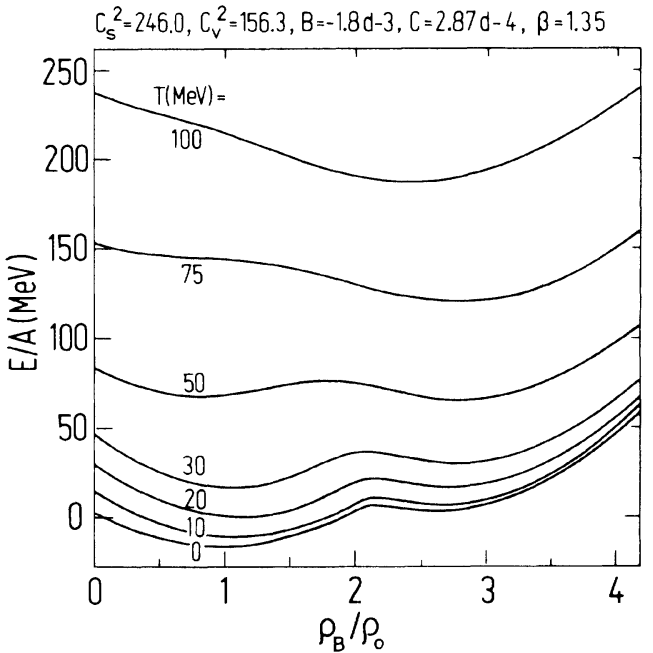

FIG. 7. Equation of state for finite temperature and $\beta=1.35$. Binding energy per nucleon vs baryon density $\rho_{B} / \rho_{0}$.

The first one at an energy density $\epsilon \simeq 100 \mathrm{MeV} / \mathrm{fm}^{3}$, and the second one at $\epsilon \simeq 400 \mathrm{MeV} / \mathrm{fm}^{3}$. These phase transitions are characterized by negative values of $c_{s}^{2}$, i.e., imaginary values of the sound velocity $c_{s}$. This indicates a mechanical instability of the system. The first phase transition at $\epsilon \cong 100 \mathrm{MeV} / \mathrm{fm}^{3}$ can be identified as the liquidvapor phase transition, as shown in Ref. 1 for the linear model. The second phase transition corresponds to the delta-density isomeric state. ${ }^{5}$ Here a baryonic phase transition from nucleonic to delta matter occurs. Solving the Rankine-Hugoniot-Taub relations for this density isomeric state the single shock solutions break down and shock instabilities occur, ${ }^{8}$ similar to those predicted ten year ago. ${ }^{14}$

The equation of state of the baryonic system and the appearance of phase transitions can be illustrated best by plotting isotherms of pressure versus the chemical potential $\mu$. In such a figure, possible phase transitions can be

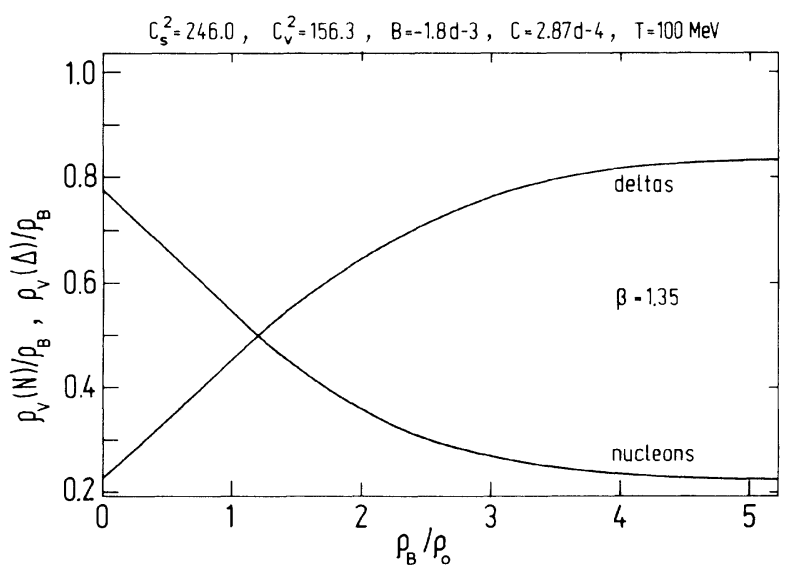

FIG. 8. Particle density of deltas and nucleons vs the baryon density for $T=100 \mathrm{MeV}$.

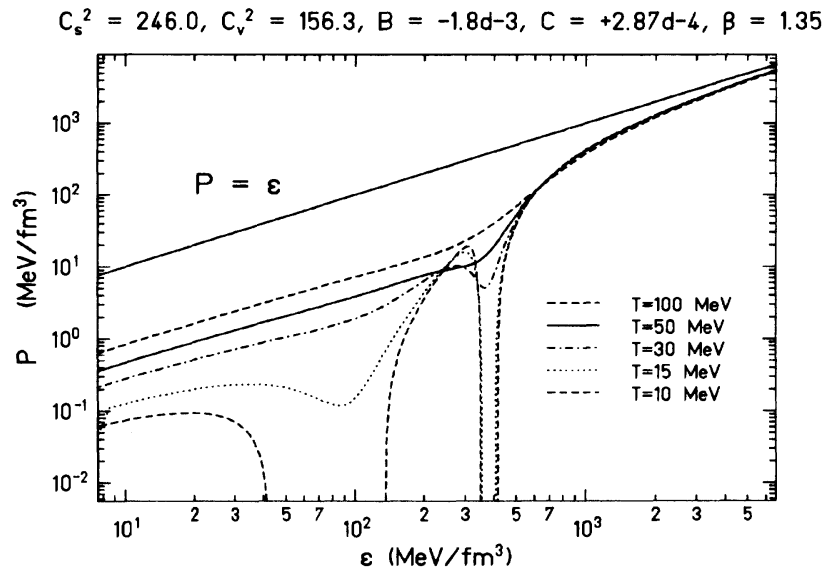

FIG. 9. Pressure vs energy density in a logarithmic scale for $\beta=1.35$ and temperatures from 10 to $100 \mathrm{MeV}$.

identified very easily: The Gibbs two phase equilibrium is established when two branches of $P(\mu, T)$ cross, i.e., thermal equilibrium $\left(T_{1}=T_{2}\right)$, chemical equilibrium $\left(\mu_{1}=\mu_{2}\right)$, and mechanical equilibrium $\left(P_{1}=P_{2}\right)$ between the two branches are automatically ensured. Observe in Fig. 10 that the isotherms separate into four regions which can be identified with nucleon-liquid, the nucleon-gas, the delta phase, and a fourth phase which corresponds to the plasma phase discussed in Ref. 1. Regions of instability develop when the incompressibility becomes negative. The van der Waals form of the equation of state in the low temperature range is due to the long range attractive forces and the short range repulsion described in the mean-field model. This first transition (liquid-gas) has a critical temperature $T_{c} \simeq 17 \mathrm{MeV}$. The critical temperature of the second phase transition is $T_{c}=41 \mathrm{MeV}$. At even high temperatures, $T \approx 130$

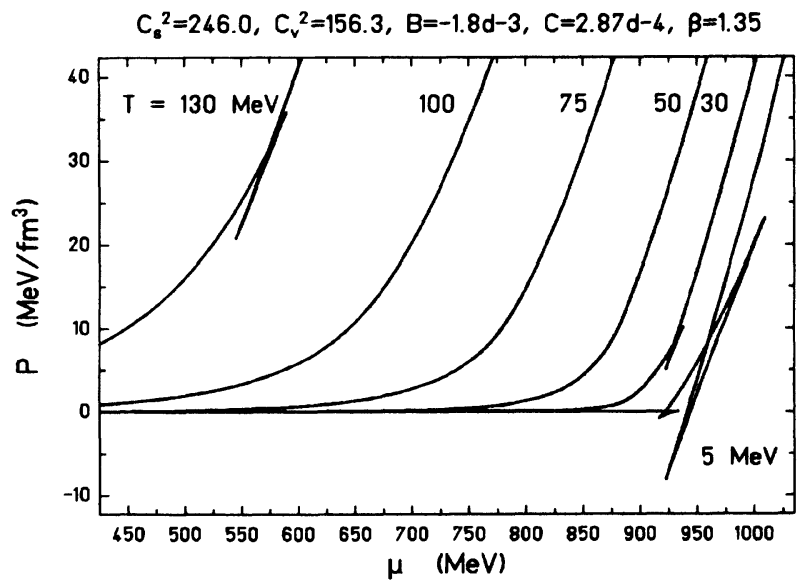

FIG. 10. Pressure $P$ vs chemical potential $\mu$ for $\beta=1.35$ and temperatures from 5 to $130 \mathrm{MeV}$. 
$\mathrm{MeV}$, an independent phase transition develops. It corresponds to the nearly massless phase of hadrons observed in Refs. 1, 4, and 6-8. This phase transition occurs not only for the vanishing chemical potential $\mu$ discussed in Ref. 1. Figure 10 shows that it exists for a higher chemical potential above $500 \mathrm{MeV}$ as well. The appearance of heavier baryons not only raises the chemical potential $\mu$, but also produces a cooling effect due to the high degeneracy factor $\gamma\left(\gamma_{\Delta}=16\right)$. This effect will be discussed in more detail for the baryonic plasma $\left(\rho_{B}=\mu=0\right)$.

Figure 11 shows the phase diagram of baryonic matter for the set of coupling constants given above and $\alpha=1$, $\beta=1.35$. We can recognize the liquid-gas coexistence, the delta matter, and the narrow (in temperature $T$ ) but broad (in density $\rho_{B} / \rho_{0}$ ) band of the transition region to the massless plasma. The liquid-vapor phase transition is not affected by the inclusion of the delta resonances, while the high-temperature phase transition is strongly shifted to lower temperatures. The three transition regions are well separated from each other.

\section{B. Critical conditions in the baryonic plasma}

Let us now explore the phase structure of the effective Lagrangian at vanishing chemical potential $\mu$, i.e., vanish-

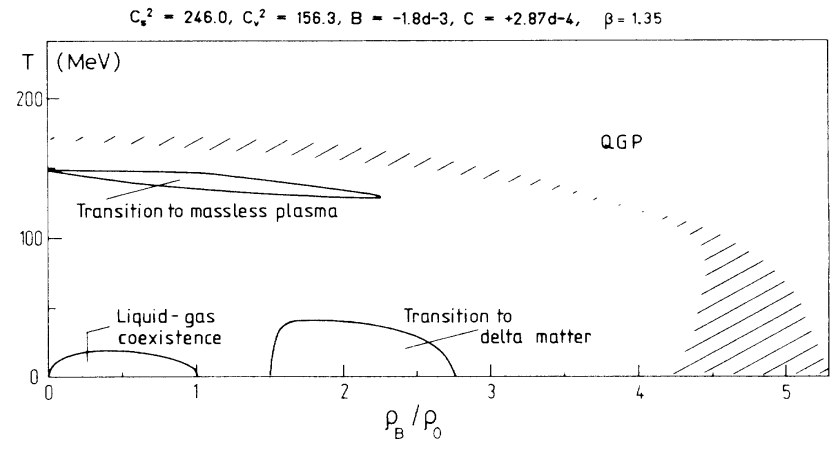

FIG. 11. Phase diagram of nuclear matter: Temperature $T$ vs baryon density $\rho_{B} / \rho_{0}$ for $\beta=1.35$ and $\alpha=1.0$. The coexistence region for liquid gas and the transition regions to delta matter and to the massless plasma are calculated. The hatched area indicates the coexistence region of quark-gluon plasma and hadronic phase. QGP stands for quark-gluon plasma.

ing vector density $\rho_{v}$. First observe that for $\mu=0$ the vector coupling constant does not appear in the thermodynamical quantities calculated in the mean field approximation. In contrast to finite $\mu$, the Fermi integration can be done analytically, yielding for the dimensionless energy density

$$
\begin{aligned}
\epsilon= & +U\left(\varphi_{0}\right)+\frac{\gamma(\mathrm{N}) T m^{* 3}(\mathrm{~N})}{\pi^{2}} \sum_{n=1}^{\infty} \frac{(-1)^{n-1}}{n}\left[K_{1}\left(z_{\mathrm{N}}\right)+\frac{3 T}{n m^{*}(\mathrm{~N})} K_{2}\left(z_{N}\right)\right] \\
& +\frac{\gamma(\Delta) T m^{* 3}(\Delta)}{\pi^{2}} \sum_{n=1}^{\infty} \frac{(-1)^{n-1}}{n}\left[K_{1}\left(z_{\Delta}\right)+\frac{3 T}{n m^{*}(\Delta)} K_{2}\left(z_{\Delta}\right)\right]
\end{aligned}
$$

and the total pressure

$$
P=-U\left(\varphi_{0}\right)+\frac{\gamma(\mathrm{N}) T^{2} m^{* 2}(\mathrm{~N})}{\pi^{2}} \sum_{n=1}^{\infty} \frac{(-1)^{n-1}}{n^{2}} K_{2}\left(z_{\mathrm{N}}\right)+\frac{\gamma(\Delta) T^{2} m^{* 2}(\Delta)}{\pi^{2}} \sum_{n=1}^{\infty} \frac{(-1)^{n-1}}{n^{2}} K_{2}\left(z_{\Delta}\right) .
$$

The self-consistency relation for the effective mass results for $\mu=0$ in

$$
\begin{aligned}
m^{*}(\mathrm{~N})=m_{\mathrm{N}}-C_{s}^{2} \frac{T}{\pi^{2}} \sum_{n=1}^{\infty} \frac{(-1)^{n-1}}{n}\{ & \gamma(\mathrm{N}) m^{* 2}(\mathrm{~N}) K_{1}\left(z_{N}\right)+\beta \gamma(\Delta) m^{* 2}(\Delta) K_{1}\left(z_{\Delta}\right) \\
& \left.+B\left[m^{*}(\mathrm{~N})-m_{\mathrm{N}}\right]^{2}+C\left[m^{*}(\mathrm{~N})-m_{\mathrm{N}}\right]^{3}\right\} .
\end{aligned}
$$

where $z(i)$ abbreviates:

$$
z(i)=-n m^{*}(i) / T
$$

for $i=\mathrm{N}, \Delta$.

In Eqs. (15)-(17), $K_{1}$ and $K_{2}$ denote the modified Bessel functions of first and second order, respectively. Figure 12 contains the solution $\mathrm{m}^{*}$ of the self-consistency equation as a function of the temperature for a linear set of coupling constants. ${ }^{15}$ The general trend of the function $m^{*}(T)$ can be understood as follows: The scalar density, which appears as a source for the scalar meson field $\varphi_{0}$, increases with increasing temperature. This leads to an increase of the mean-field value of $\varphi_{0}$. Since the scalar meson describes an attractive interaction of the nucleons, these will be bound more strongly, and thus the effective mass is reduced. This mechanism is reinforced by the fact that a decrease of the effective mass increases the scalar density again. One striking feature is the sudden drop in $m^{*}$ at temperature $T \simeq 200 \mathrm{MeV}$, where the transition from $m^{*}=0.75$ to $m^{*}=0.25$ occurs in an interval of $\Delta T \simeq 5 \mathrm{MeV}$ only. The critical temperature of the phase transition decreases to $T=100-150 \mathrm{MeV}$ if we take the delta resonances into account. This is the cooling effect mentioned above and is seen similarly in shock calculations. $^{8}$ It also depends on the ground-state properties 


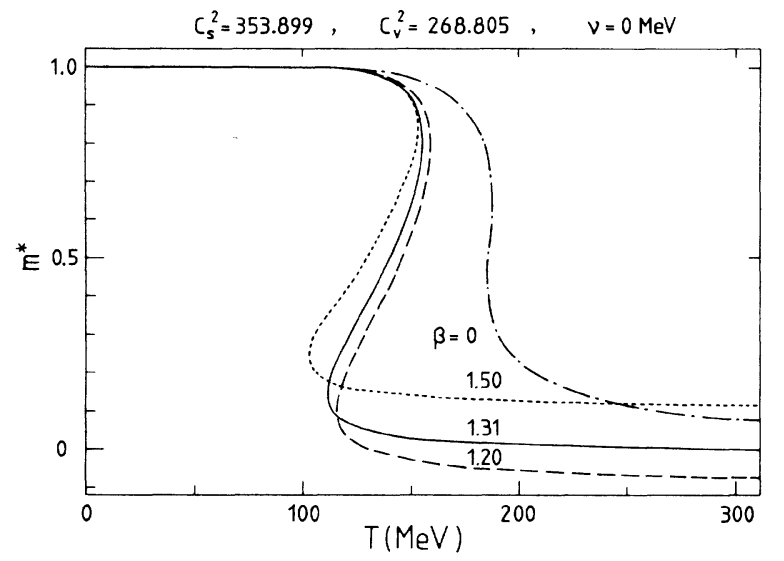

FIG. 12. Effective mass $m^{*} / m$ vs temperature $T$ for vanishing chemical potential $\mu$ and $\beta=0,1.2,1.31,1.5$.

used. The mathematical structure of the self-consistency equation is so simple that is is possible to understand how this decoupling happens. First we remark that the specific heat is linear in $\partial m^{*} / \partial T$. So whenever we see a sudden fall in $m^{*} /(T)$, there is a peak in the specific heat. In addition, if we have three solutions of the self-consistency equation, similarly the total energy density in that region is also triple valued. The fact that we see one or two poles or only a peak in $\partial m^{*} / \partial T$ means, respectively, that we have a phase transition of second or first order or continuous thermodynamical behavior. The most important point is the occurrence of negative effective nucleon masses evaluated for $\alpha=1$ and $\beta=1.20$. When the effective mass vanishes, there is no energy gap between the baryons and the antibaryons. Thus spontaneous particle production can occur. In the linear model an analytic solution for the high-temperature behavior of the effective masses can be derived:

$$
\begin{aligned}
& \frac{m^{*}(\mathrm{~N})}{m_{\mathrm{N}}} \underset{T \rightarrow \infty}{\longrightarrow}-\beta \gamma(\Delta)\left(\frac{m_{\Delta}}{m_{\mathrm{N}}}-\beta\right) \times \text { const }, \\
& \frac{m^{*}(\Delta)}{m_{\mathrm{N}}} \underset{T \rightarrow \infty}{\longrightarrow}+\gamma(\mathrm{N})\left(\frac{m_{\Delta}}{m_{\mathrm{N}}}-\beta\right) \times \text { const } .
\end{aligned}
$$

It is obvious that the sign of the effective masses depends on the sign of the quantity inside the large parentheses, but in an opposite way. So we have three different possibilities in choosing $\beta$. The first one is to choose $\beta>1.31$, so that we get a positive effective nucleon mass, but then the effective delta mass becomes negative. The second one is to choose $\beta<1.31$. Then we will get a positive effective delta mass, but have to cope with negative effective nucleon mass. The third possibility is $\beta \simeq 1.31$, as we had discussed analytically for the baryonic plasma, in which case both effective masses will go to zero in the high temperature regime, i.e., we will get chiral symmetry. This only happens if $\beta$ is equal to the ratio of the delta mass to the nucleon mass [Eq. (14)]. This result is only valid for the scalar coupling constant, because the vector meson does not contribute to the effective masses. Thus the only way to get rid of the unrealistic negative masses is to choose $\beta=m(\Delta) / m(\mathrm{~N}) \simeq 1.31$, so that both effective masses converge to zero for $T \rightarrow \infty$. This requirement does not imply that $\beta$ should be chosen in this way in all mean-field calculations: The extrapolation to high $T$ must certainly be questioned for the present approach. It must be kept in mind that this picture is the result of a mean-field approximation. Hence the detailed structure of the phase transitions will certainly be different in the full quantum field theory. Another remark concerns the observation that at high temperature the system behaves like an almost free zero mass fermion gas with a constant shift in the energy density and in the pressure. This is quite analogous to the expected chiral phase transition in high temperature quantum chromodynamics (QCD). However, there is no liberation of internal degrees of freedom of hadrons in the linear mean-field model.

Summarizing this section we may state that around the temperature $T \simeq 100-200 \mathrm{MeV}$ the pressure and the internal energy become, up to a constant, those of a free massless fermion gas having the degeneracy factor of nuclear matter. We interpret these results such that the nuclear field theory, as a low temperature effective theory of hadronic matter, indicates the occurrence of a sudden change in the thermodynamical behavior around $T=100-200 \mathrm{MeV}$ at zero baryon density, in some analogy to quark deconfinement in lattice QCD calculations. The last result is that this phase transition occurs for different $\beta(\beta=1.2-1.5)$, and in the special case $\beta \simeq 1.31$ for all possible values of $C_{s}^{2}$. While this phase transition was parameter dependent in the normal linear and nonlinear mean-field theory (Ref. 1), it is now always present. This is due to the delta-scalar coupling which reduces the effective nucleon mass very strongly. The critical value for $\beta=1.35$ is $C_{s}^{2}=20$. For such a low scalar coupling one obtains an effective nucleon mass above 0.9 for the ground state or a compressibility of $2000 \mathrm{MeV}$, which are both much too high.

\section{CONCLUSION}

A detailed analysis of the phase structure in the nonlinear mean field model including delta resonances shows that with parameters which reproduce the properties of ground state nuclear matter, the model predicts a lowtemperature nucleonic liquid-vapor phase transition at $T_{c} \simeq 15-20 \mathrm{MeV}$, and another one at temperatures above $100 \mathrm{MeV}$ connected with the critical conditions in the high-temperature baryon, antibaryon plasma. These transition are quite similar to those found in the linear meanfield model, but the critical temperature $T_{c}$ in the hightemperature regime is reduced. The location of this hightemperature phase transition is strongly parameter dependent. For a system of nucleons and delta resonances, we do observe this state for all coupling constants which describe nuclear ground-state properties. Thus the inclusion of nonlinear scalar interactions or delta-meson couplings into the mean-field Lagrangian does not alter this critical 
behavior of the model. Additional problems enter by a third phase transition, which results from the delta interaction with the meson fields. The first problem is the nucleon-delta phase transition at $T_{c} \leq 45 \mathrm{MeV}$, and the second one the creation of negative effective masses for $\beta \neq m(\Delta) / m(\mathbf{N})$. One can only get rid of this unrealistic behavior by demanding the scalar-delta coupling to be proportional to the mass ratio. This can yield delta iso- mers for certain choices of the vector coupling constant $(\alpha<\beta)$.

\section{ACKNOWLEDGMENT}

We thank Dirk Rischke for allowing us to use his results concerning the phase transition from quark gluon plasma to hadronic matter (Fig. 11).
${ }^{1}$ J. Theis, G. Graebner, G. Buchwald, J. A. Maruhn, W. Greiner, H. Stöcker, and J. Polonyi, Phys. Rev. D 28, 2286 (1983).

2J. D. Walecka, Ann. Phys. 83, 491 (1974); B. Serot and J. D. Walecka, Advances in Nuclear Physics (Plenum, New York, 1985).

${ }^{3}$ J. Boguta and A. R. Bodmer, Nucl. Phys. 292A, 413 (1977); J. Boguta and H. Stöcker, Phys. Lett. 120B, 289 (1983); J. Boguta, ibid. 106B, 250 (1981).

${ }^{4}$ S. I. A. Garpman, N. K. Glendenning, and Y. J. Karant, Nucl. Phys. 322A, 382 (1979).

5 J. Boguta, Phys. Lett. 109B, 251 (1982).

${ }^{6}$ T. Nakai and S. Takagi, Prog. Theor. Phys. 71, 1188 (1984).

${ }^{7}$ N. K. Glendenning, Phys. Lett. 144B, 158 (1984).

${ }^{8}$ B. Waldhauser, J. Theis, J. A. Maruhn, H. Stöcker, and W. Greiner, Gesellschaft für Schwerionenforschung Darmstadt m.b.H. Report ISSN 0174-0814 1985, pp. 167 and 168; B. Waldhauser, Diplomathesis, Institut für Theoretische Physik der Johann-Wolfgang-Goethe Universität, Frankfurt am
Main, 1986.

${ }^{9}$ P. Levai, B. Lukacs, B. Waldhauser, and J. Zimanyi, Phys. Lett. 177B, 5 (1986).

${ }^{10}$ H. Stöcker, W. Greiner, and W. Scheid, Z. Phys. A 286, 121 (1978).

${ }^{11}$ R. Stock, R. Bock, R. Brockmann, J. W. Harris, A. Sandoval, H. Ströbele, K. L. Wolf, H. G. Pugh, L. S. Schröder, R. E. Renfordt, A. Dacal, and M. E. Ortiz, Phys. Rev. Lett. 49, 1236 (1982); J. W. Harris, R. Stock, R. Bock, R. Brockmann, A. Sandoval, H. Ströbele, G. Odyniec, H. G. Pugh. L. S. Schröder, R. E. Renfordt, D. Schall, D. Bangert, W. Rauch, and K. L. Wolf, Phys. Lett. 153B, 377 (1985).

${ }^{12}$ D. Hahn and H. Stöcker, Nucl. Phys. A 452, 723 (1986).

${ }^{13}$ H. Kruse, B. V. Jacak, and H. Stöcker, Phys. Rev. Lett. 54, 289 (1985).

${ }^{14}$ J. Hofmann, H. Stöcker, U. Heinz, W. Scheid, and W. Greiner, Phys. Rev. Lett. 36, 88 (1976).

${ }^{15}$ P.-G. Reinhard, M. Rufa, J. Maruhn, W. Greiner, and J. Friedrich, Z. Phys. A 323, 13 (1986). 